\title{
Research on Algebraic Configuration Description Method and kinematical modeling for Modular Robot
}

\author{
Xiao Li ${ }^{1, a}$, Hanxu Sun ${ }^{2, b}$, Linjing Liao ${ }^{2, \mathrm{c}}$, Jingzhou Song ${ }^{2, \mathrm{~d}}$ \\ ${ }^{1}$ School of Mechanical Engineering and Automation, Beihang University, in China \\ ${ }^{2}$ School of Automation, Beijing University of Posts and Telecommunication, in China

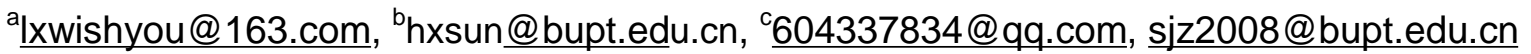

Key words: Modular robot, Configuration Description Method, Kinematics.

Abstract. We propose a new algebraic configuration description method (ACDM) for the modular robot. The ACDM can describe each mechanical configuration in the robotic configuration parameters, and make up for the inadequacy of $\mathrm{D}-\mathrm{H}$ method in multiple configuration. The algebraic configuration description method support complicated calculation about the parameters in joint level. The configuration algebraic expression and kinematic model are established in this paper for the modular robot. And kinematical modeling and simulation results show that the algebraic configuration description method is effective.

\section{Introduction}

Modular robot has short time and high efficient in development. The design of robot can be de divided into the design of modules and the design of robot's configuration, the former is much easier than the latter so that it needs less time. When the configuration of robot needs to change, our main task is to do the configuration design and some special modules design, which greatly improves the efficient of design. Many institutions have made research about modular robot, such as the RMMS in Carnegie Mellon University[1,2], the modular robot system MRS in university of Toronto[3], the PowerCube series module products in the AMTEC company of Germany[4-6] .

Reconfigurable modular robot system(RMRS) consists of a series of different functions, characteristics and assembly function of the standard size of joint or link modules, the way to the building blocks of robotic systems that have been assembled[7]. This combination is not a simple mechanical restructuring, including control system, electronic hardware, control algorithm, software, such as restructuring. Because the modular joint itself is a set of drive, driving, control, communication as a whole unit[8].

The D-H method has been widely applied in kinematic model. But the D-H method is inefficient when modeling in multiple configuration. So the new algebraic configuration description method $(\mathrm{ACDM})$ is proposed for the modular robot in this paper.

\section{The modular robot parameters}

The design configuration of 6-DOF modular reconfigurable robot is shown in Figure 1. And it has 6 DOF. The joint weight $(\mathrm{m} 1 \sim \mathrm{m} 6)$ is $13.00 \mathrm{~kg}, 13.0 \mathrm{~kg}, 8.5 \mathrm{~kg}, 8.5 \mathrm{~kg}, 5.5 \mathrm{~kg}, 5.5 \mathrm{~kg}$. The effective length of link module is $100 \mathrm{~mm}$, and its weight is $0.8 \mathrm{~kg}$. Because of the symmetrical design, barycentric position of the joints are all in the geometric center.

The basic module consists of three kinds of joints: Type 1, Type 2 and Type 3 . The joints can also be designed in accordance with the requirements into larger or smaller Type. The appearance size of Type 1 is $146^{*} 146 * 293 \mathrm{~mm}$, the appearance size of Type 2 is $110^{*} 110 * 221 \mathrm{~mm}$, and the appearance size of Type 3 is $90 * 90 * 181 \mathrm{~mm}$. Among them Joint 1 and Joint 2 are Type1, Joint 3 and Joint are Type2, Joint 5 and Joint 6 are Type3. The configuration of joints module are shown in Figure 2.

The Joints design parameters are shown in Table 1. We can see the Joints support flexible way of refactoring. 


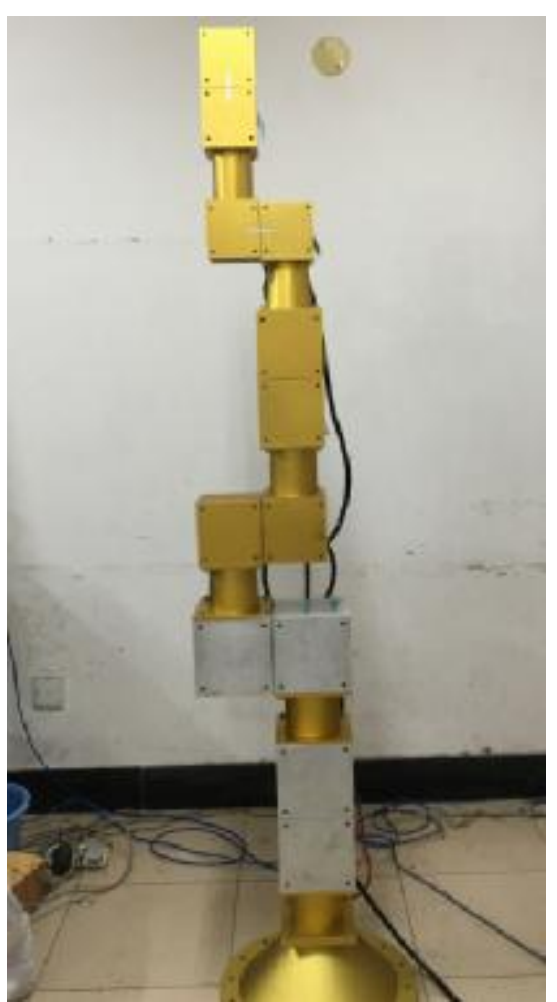

Figure 1 The 6-DOF modular robot

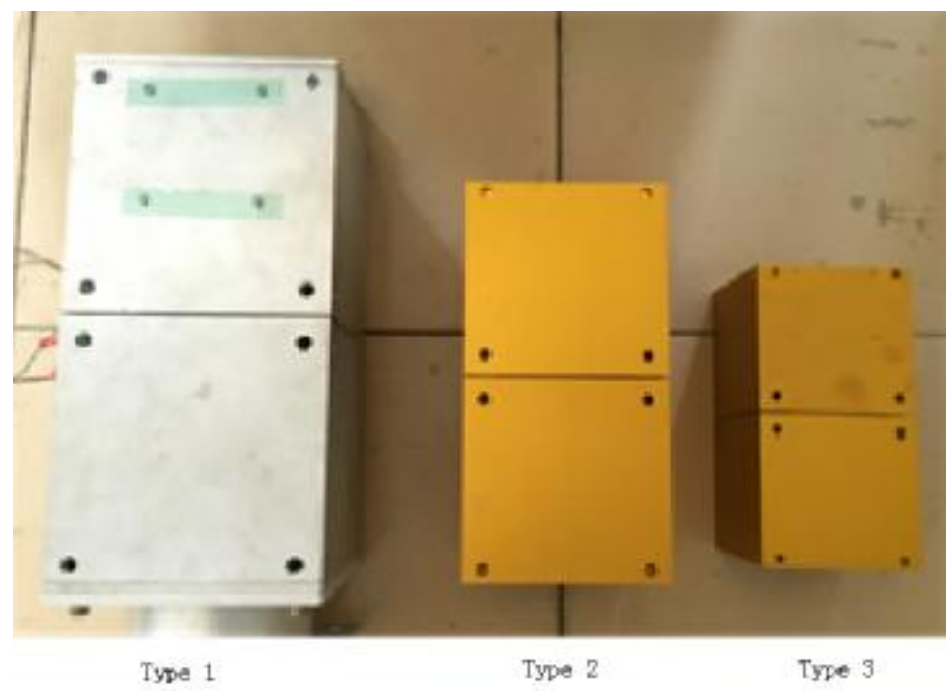

Figure 2 The Type of modular joints

Table 1 Joint design parameters

\begin{tabular}{ccccc}
\hline Designation & Unit & Type 1 & Type 2 & Type 3 \\
\hline Rated torque & $\mathrm{Nm}$ & 580 & 220 & 100 \\
\hline Peak torque & $\mathrm{Nm}$ & 1300 & 750 & 420 \\
\hline Rotation angle & $\circ$ & $>360$ & $>360$ & $>360$ \\
\hline Weight & $\mathrm{kg}$ & 13 & 8.5 & $\leq .5$ \\
\hline Repeated accuracy & $\circ$ & $\leq 0.002$ & 70.002 & $\leq 0.002$ \\
\hline $\begin{array}{c}\text { maximum angular } \\
\text { velocity }\end{array}$ & $\mathrm{rpm}$ & 35 & 70 & 80 \\
\hline reduction ratio & 1 & $160 / 120$ & $160 / 120$ & $160 / 120 / 100$ \\
\hline nominal voltage & VDC & 48 & 48 & 48 \\
\hline \multirow{2}{*}{ electrical interface } & & RS232;CAN-Open; & RS232;CAN-Open; & RS232;CAN-Open; \\
& & USB & USB & USB \\
\hline
\end{tabular}

\section{The Algebraic Configuration Description Method}

The Joint can combination the type of serial robots, parallel robot, mixed type and complex robot for the Reconfigurable modular robot. According to the connection mode, the configuration described in the following definition:

Common base model: the rotary joints is defined as $R_{y}^{x}$, the displacement of the joint is defined as $D_{y}^{x}$, the connecting rod is described as $L_{y}^{x}$.

And the $\mathrm{x}$ is position number, the $\mathrm{y}$ is in combination with surface code.

And the $\mathrm{x}$ or $\mathrm{y}$ be omit when robot has one joint.

The expression of configuration: The Joints (Joint or link) is defined as $N_{x}$, the robot is defined as $N_{0}$. When it has child-robot, the child-robot is defined as $N_{O X}$.

The joint of multiple DOF is defined as multiplication cross: $N_{x}=N_{1} \times N_{2}$.

The series robot is described as dot product: $N_{x}=N_{1} \cdot N_{2}$.

The parallel robot is described as sum: $N_{x}=N_{1}+N_{2}$

The connecting rod assignment for the joints when is has the function of special. The connecting 
rod is is described as: $N_{x}=L_{y}^{x}$.

\section{Configuration expression and kinematic model}

The configuration expression of the modular robot in Figure 1 can be expressed as following Formula (1):

$$
N_{x}=N_{1} \cdot N_{2} \cdot N_{3} \cdot N_{4} \cdot N_{5} \cdot N_{6}
$$

The $\mathrm{N}_{1}$ to $\mathrm{N}_{6}$ is corresponding to Joint 1 to Joint 6 . The kinematic model is corresponding to each matrix of the Joint.

The Joint transformation matrix is expressed in Equation (2).

$$
{ }_{\mathrm{i}}^{\mathrm{i}} \mathrm{T}=\left[\begin{array}{cccc}
c \theta_{\mathrm{i}} & -s \theta_{\mathrm{i}} & 0 & \mathrm{a}_{\mathrm{i}-1} \\
\mathrm{~s} \theta_{\mathrm{i}} c \alpha_{\mathrm{i}-1} & c \theta_{\mathrm{i}} c \alpha_{\mathrm{i}-1} & -s \alpha_{\mathrm{i}-1} & -\mathrm{d}_{\mathrm{i}} s \alpha_{\mathrm{i}-1} \\
s \theta_{\mathrm{i}} s \alpha_{\mathrm{i}-1} & c \theta_{\mathrm{i}} s \alpha_{\mathrm{i}-1} & c \alpha_{\mathrm{i}-1} & \mathrm{~d}_{\mathrm{i}} c \alpha_{\mathrm{i}-1} \\
0 & 0 & 0 & 1
\end{array}\right]
$$

The D-H parameters are shown in Table 2.

Table 2 The D-H parameters for 6-DOF robot

\begin{tabular}{ccccc}
\hline Link $\mathrm{i}$ & $\boldsymbol{\alpha}_{\mathrm{i}-1}$ & $/ \mathrm{mm}$ & $\mathrm{d}_{\mathrm{i}}$ & $\theta_{\mathrm{i}}$ \\
\hline 1 & 0 & 0 & 420 & 0 \\
\hline 2 & $\mathrm{pi} / 2$ & 0 & 0 & $\mathrm{pi} / 2$ \\
\hline 3 & $\mathrm{pi}$ & 254 & 0 & $\mathrm{pi} / 2$ \\
\hline 4 & $\mathrm{pi} / 2$ & 0 & 469 & 0 \\
\hline 5 & $\mathrm{pi} / 2$ & 0 & 111 & 0 \\
\hline 6 & $-\mathrm{pi} / 2$ & 0 & 251 & 0 \\
\hline
\end{tabular}

The transformational matrix are expressed in Equation (3) to Equation (8)

$$
\begin{aligned}
& A_{1}=\left[\begin{array}{cccc}
c \theta_{1} & -s \theta_{1} & 0 & 0 \\
s \theta_{1} & c \theta_{1} & 0 & 0 \\
0 & 0 & 1 & 420 \\
0 & 0 & 0 & 1
\end{array}\right] \\
& A_{2}=\left[\begin{array}{cccc}
c \theta_{2} & -s \theta_{2} & 0 & 0 \\
0 & 0 & -1 & 0 \\
s \theta_{2} & c \theta_{2} & 0 & 0 \\
0 & 0 & 0 & 1
\end{array}\right] \\
& A_{3}=\left[\begin{array}{cllc}
c \theta_{3} & -s \theta_{3} & 0 & 254 \\
-s \theta_{3} & -c \theta_{3} & 0 & 0 \\
0 & 0 & -1 & 0 \\
0 & 0 & 0 & 1
\end{array}\right] \\
& \mathrm{A}_{4}=\left[\begin{array}{cccc}
\mathrm{c} \theta_{4} & -\mathrm{s} \theta_{4} & 0 & 0 \\
0 & 0 & -1 & -469 \\
\mathrm{~s} \theta_{4} & c \theta_{4} & 0 & 0 \\
0 & 0 & 0 & 1
\end{array}\right] \\
& A_{5}=\left[\begin{array}{cccc}
c \theta_{5} & -s \theta_{5} & 0 & 0 \\
0 & 0 & -1 & -111 \\
s \theta_{5} & c \theta_{5} & 0 & 0 \\
0 & 0 & 0 & 1
\end{array}\right] \\
& A_{6}=\left[\begin{array}{cccc}
c \theta_{6} & -s \theta_{6} & 0 & 0 \\
0 & 0 & 1 & 251 \\
-s \theta_{6} & -c \theta_{6} & 0 & 0 \\
0 & 0 & 0 & 1
\end{array}\right]
\end{aligned}
$$

So kinematic model of the 6-DOF robot is expressed in Equation (9) as following.

$$
T=\left[\begin{array}{cccc}
c \theta_{1} & -s \theta_{1} & 0 & 0 \\
s \theta_{1} & c \theta_{1} & 0 & 0 \\
0 & 0 & 1 & 420 \\
0 & 0 & 0 & 1
\end{array}\right]\left[\begin{array}{cccc}
c \theta_{2} & -s \theta_{2} & 0 & 0 \\
0 & 0 & -1 & 0 \\
s \theta_{2} & c \theta_{2} & 0 & 0 \\
0 & 0 & 0 & 1
\end{array}\right]\left[\begin{array}{cccc}
c \theta_{3} & -s \theta_{3} & 0 & 254 \\
-s \theta_{3} & -c \theta_{3} & 0 & 0 \\
0 & 0 & -1 & 0 \\
0 & 0 & 0 & 1
\end{array}\right] .
$$




$$
\left[\begin{array}{cccc}
c \theta_{4} & -s \theta_{4} & 0 & 0 \\
0 & 0 & -1 & -469 \\
s \theta_{4} & c \theta_{4} & 0 & 0 \\
0 & 0 & 0 & 1
\end{array}\right]\left[\begin{array}{cccc}
c \theta_{s} & -s \theta_{5} & 0 & 0 \\
0 & 0 & -1 & -111 \\
s \theta_{5} & c \theta_{5} & 0 & 0 \\
0 & 0 & 0 & 1
\end{array}\right]\left[\begin{array}{cccc}
c \theta_{6} & -s \theta_{6} & 0 & 0 \\
0 & 0 & 1 & 251 \\
-s \theta_{6} & -c \theta_{6} & 0 & 0 \\
0 & 0 & 0 & 1
\end{array}\right]
$$

Thus the $\mathrm{N}_{1}=\mathrm{A}_{1}, \mathrm{~N}_{2}=\mathrm{A}_{2}, \mathrm{~N}_{3}=\mathrm{A}_{3}, \mathrm{~N}_{4}=\mathrm{A}_{4}, \mathrm{~N}_{5}=\mathrm{A}_{5}, \mathrm{~N}_{6}=\mathrm{A}_{6}$. The kinematics parameters can be calculated directly in ACDM.

\section{Conclusions}

In this paper, we propose a new algebraic configuration description method for the modular robot. And the algebraic configuration description method (ACDM) can describe all mechanical configuration in the robot configuration parameters, and the ACDM make up for the inadequacy of $\mathrm{D}-\mathrm{H}$ method in multiple configuration. The ACDM support complicated calculation about the parameters in joint level. The configuration algebraic expression and kinematic model are established in this paper for modular robot. And the kinematical modeling and simulation results show that the algebraic configuration description method is effective.

\section{Acknowledgements}

This work was financially supported by the School of Automation, Beijing University of Posts and Telecommunication, in China.

\section{References}

[1] Schmitz D, Khosla P, Kanade T. The CMU Reconfigurable Modular Manipulator System[C]// TECHNICAL REPORT. 2010.

[2] Stewart D B, Khosla P K. Rapid development of robotic applications using component-based real-time software[C]// Ieee/rsj International Conference on Intelligent Robots and Systems 95. 'human Robot Interaction and Cooperative Robots', Proceedings. IEEE, 1995:465.

[3] Cohen R, Lipton M G, Dai M Q, et al. Conceptual Design of a Modular Robot[J]. Journal of Mechanical Design, 1992, 114(1):117-125.

[4] Smith C, Christensen H I. Using COTS to Construct a High Performance Robot Arm[C]// Robotics and Automation, 2007 IEEE International Conference on. IEEE, 2007:4056 - 4063.

[5] Escudero S, Luis J. Control cinemático y de fuerza de una mano robótica para el agarre estable[J]. 2015.

[6] Macanás Valera J. Interacción entre webcam y brazo robot para el posicionamiento del efector final[J]. 2015.

[7] Brock O, Trinkle J, Ramos F. Distributed Localization of Modular Robot Ensembles[M]// Robotics:Science and Systems IV. MIT Press, 2010:33-40.

[8] Deng J, Wang P, Meng Q, et al. Design and Development on a Reconfigurable Modular Joint of the Robot Arm[M]// Advances in Reconfigurable Mechanisms and Robots II. 2016.

[9] Chen I M. Rapid response manufacturing through a rapidly reconfigurable robotic workcell[J]. Robotics and Computer-Integrated Manufacturing, 2001, 17(3):199-213. 